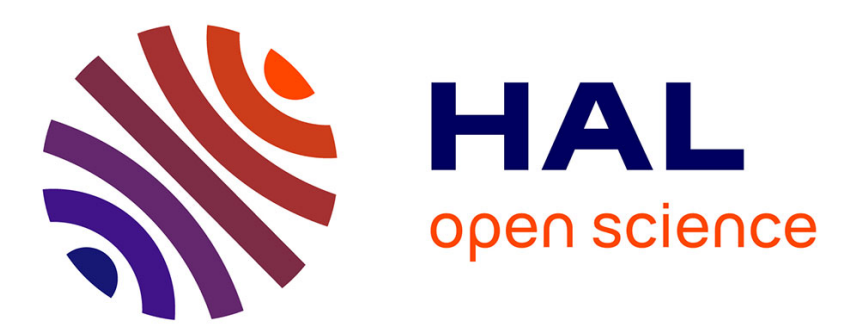

\title{
Bounded Control of a General Extended Chained Form Systems
}

\author{
Ahmad Hably, Nicolas Marchand
}

\section{To cite this version:}

Ahmad Hably, Nicolas Marchand. Bounded Control of a General Extended Chained Form Systems. CDC 2014 - 53rd IEEE Conference on Decision and Control, Dec 2014, Los Angeles, United States. 6 p. hal-01067123

\section{HAL Id: hal-01067123 https://hal.science/hal-01067123}

Submitted on 23 Sep 2014

HAL is a multi-disciplinary open access archive for the deposit and dissemination of scientific research documents, whether they are published or not. The documents may come from teaching and research institutions in France or abroad, or from public or private research centers.
L'archive ouverte pluridisciplinaire HAL, est destinée au dépôt et à la diffusion de documents scientifiques de niveau recherche, publiés ou non, émanant des établissements d'enseignement et de recherche français ou étrangers, des laboratoires publics ou privés. 


\section{Bounded Control of a General Extended Chained Form Systems}

\author{
Ahmad Hably* \\ GIPSA-lab, Grenoble-inp \\ Saint-Martin d'Heres, France
}

\author{
Nicolas Marchand** \\ GIPSA-lab, CNRS \\ Saint-Martin d'Heres, France
}

\begin{abstract}
In this paper, a state feedback control is proposed for the control of second-order chained form system with bounded inputs. The feedback law is based on a receding horizon strategy that provides convergence of the system to any desired final state. Numerical simulations are given to show the effectiveness of the proposed control strategy.
\end{abstract}

\section{INTRODUCTION}

In this paper a control law is developed for the general second order chained-form system defined as:

$$
\left\{\begin{array}{l}
\ddot{x}_{0}=u \\
\ddot{x}_{1}=v \\
\ddot{x}_{i}=x_{i-1} u \quad i \in\{2, \ldots, m\}
\end{array}\right.
$$

where $x_{0, \ldots, m}$ are the states and $u, v$ are the control inputs. Controlling this type of system is not easy because it does not meet the Brockett conditions [3]. These conditions are necessary for the existence of static state feedback control $\mathrm{C}^{1}$. In addition, the second order chained system differs from first-order chained systems, initially proposed by [11] as it contains a drift component. For these reasons, system (1) has attracted much attention during recent years in the nonlinear control community. In [7], a discontinuous control to exponentially stabilize the generalized version of system (1) is presented. It was also the subject of study in [12] where the exponential stability is guaranteed by applying a continuous control law variable over time. Furthermore, [13] has studied the convergence of system (1) by a state and output feedback based on sampled data control.

In the case where $i=2$, one gets the following system:

$$
\left\{\begin{array}{l}
\ddot{x}_{0}=u \\
\ddot{x}_{1}=v \\
\ddot{x}_{2}=x_{1} u
\end{array}\right.
$$

Typical examples of this system include unicycle-type vehicles, car-like vehicles and planar underactuated manipulators. The V/STOL aircraft without gravity [6] can also be transformed into a system that is equivalent to the second-order chained form using coordinate and feedback transformation [2]. This particular system has been studied by several authors. In particular, [4] has presented conditions for system (2) to be linearizable by a state transformation and discontinuous control . In [2], a Lyapunov-type stability and origin exponential convergence of system (2) were provided by a continuous time-variable homogeneous control law. The work in [10] has studied the practical stabilization of a

*Corrseponding author

Ahmad.Hably@grenoble-inp.fr

**Nicolas.Marchand@gipsa-lab. fr class of constrained systems that includes the chained-form systems by applying a transverse function approach while [8] has applied the vertical transverse as an extension transverse function approach for the second order systems.

Despite the rich literature on the subject, the control problem of system (1) that respects saturation constraints on the control inputs is not explicitly addressed. This problem is only treated for first order chained systems by a discontinuous approach proposed in [9] and a predictive control proposed in [1].

This actual paper can be considered as a generalization of [1] in the case of second order systems with saturation constraints. In addition, it generalizes our previous work [5] where we only considered a chained-form system of length 2. This paper addresses systems with an arbitrary chain length where a new choice for the basis functions of the control is to be applied. It turns out that this generalization is in no way straightforward. The proposed control is based on the principle of predictive control where feedback is obtained by solving at each sampling instant an open-loop optimization problem in which the current state $x(k)$ plays the role of initial state. Then the first part of the optimal control sequence is applied. At the next sampling instant $k+1$, a new open-loop optimization problem is solved with $x(k+1)$ as the initial state, the first part of the resulting optimal sequence again is applied and so on.

The paper is organized as follows. In section II, the formulation of the open loop control is given. In section III, two algorithms are provided to find suboptimal solutions of the optimization problem. In section IV, the state feedback control to be applied in closed-loop is formulated. Some numerical simulations are presented in section V. The paper ends with conclusions in section VI.

Notation: In this paper, $\delta>0$ denotes some fixed sampling period. For any time-dependent signal $w(),. w(k)$ simply denotes $w(k \delta)$. The classical notation $X\left(t ; 0 ; \chi_{0} ; w\right)$ is used to denote the solution at time instant $t$ of the system starting from an initial state $\chi_{0}$ at initial time $t=0$ under the control $w($.$) .$

\section{OPEN LOOP CONTROL FORMULATION}

System (1) can be divided into two subsystems. The first sub-system $\Sigma_{1}$ is linear and takes the form of a double integrator with $u$ the control and $\zeta=\left(x_{0}, \dot{x}_{0}\right)^{T}$ the state vector. The second sub-system $\Sigma_{2}$ is linear, variable in time, if $u$ is taken as a function of time, with $v$ the control and $z=\left(x_{m}, \dot{x}_{m}, \ldots, x_{1}, \dot{x}_{1}\right)^{T}$ the state vector. The global state 
vector of system (1) is $y=[\zeta z]^{T}$. The open loop piecewise continuous control sequence is then defined by:

$$
W=\left(\begin{array}{c}
U \\
V
\end{array}\right)=\left(\left(\begin{array}{l}
u_{0} \\
v_{0}
\end{array}\right),\left(\begin{array}{l}
u_{1} \\
v_{1}
\end{array}\right), \ldots,\left(\begin{array}{c}
u_{n-1} \\
v_{n-1}
\end{array}\right)\right)
$$

where $u_{i}$ and $v_{i}$ represent the control inputs applied to the system at time $i$ to direct system's state $y_{i}$ to $y_{i+1}$. The time horizon with a constant sampling period $\delta$ is given by $\tilde{t}=\left(\begin{array}{llll}0 & \delta & \ldots & n \delta\end{array}\right)$.

The ultimate goal of this command is to steer the system (1) to the desired final state $y_{d}=\left(\zeta_{d}, z_{d}\right)^{T}$ starting from the initial state $y_{0}=\left(\zeta_{0}, z_{0}\right)^{T}$. In the following, a clear parametrization of (3) is given.

1) Profile of $U$ : Subsystem $\Sigma_{1}$ is expressed by the following equation:

$$
\Sigma_{1}: \dot{\zeta}=A_{\Sigma_{1}} \zeta+B_{\Sigma_{1}} u=\left(\begin{array}{ll}
0 & 1 \\
0 & 0
\end{array}\right) \zeta+\left(\begin{array}{l}
0 \\
1
\end{array}\right) u .
$$

One choice of $u_{i}$ can be made by taking the following linear form in $i=0, \ldots, n-1$

$$
u_{i}=\alpha i+\beta=\left[\begin{array}{ll}
i & 1
\end{array}\right]\left[\begin{array}{l}
\alpha \\
\beta
\end{array}\right]
$$

where $\alpha$ and $\beta$ are functions of the initial state $\zeta_{0}$ and final state $\zeta_{n}$. One method to find these parameters is to sample $\Sigma_{1}$ over the entire prediction horizon stating from the initial state $\zeta_{0}$. State matrix $A_{1}$ and command matrix $B_{1}$ of $\Sigma_{1}$, obtained by sampling, take the following form as a function of the sampling period $\delta$

$$
A_{1}=\left[\begin{array}{ll}
1 & \delta \\
0 & 1
\end{array}\right] ; \quad B_{1}=\left[\begin{array}{c}
\frac{\delta^{2}}{2} \\
\delta
\end{array}\right]
$$

By applying the corresponding control $u_{i}, \Sigma_{1}$ will reach to the final state $\zeta_{n}$ starting from $\zeta_{0}$ :

$$
\zeta_{n}=A_{1}^{n} \zeta_{0}+\sum_{i=0}^{n-1}\left(A_{1}\right)^{n-1-i} B_{1} u_{i}
$$

Using the definition $u_{i}$ (5), the final state (7) after $n$ sampling periods can be written as:

$$
\zeta_{n}=A_{1}^{n} \zeta_{0}+\Gamma_{n}\left[\begin{array}{c}
\alpha \\
\beta
\end{array}\right]
$$

with the square matrix

$$
\Gamma_{n}=\sum_{i=0}^{n-1}\left(A_{1}\right)^{n-1-i} B_{1}\left[\begin{array}{ll}
i & 1
\end{array}\right]
$$

In the following section, we will present the necessary conditions for the existence of the piecewise continuous control $U$.

2) Existence of $U$ : To fulfill our objective to steer subsystem $\Sigma_{1}$ from the initial state $\zeta_{0}$ to the final state $\zeta_{n}$, equal to the desired state $\zeta_{d}$, in $n$ sampling periods, $\Gamma_{n}$ must be of full rank which is a result of the following lemma.

Lemma 1: $\forall n>1$, the square matrix $\Gamma_{n}$ is of full rank. Proof: see appendix I.
By applying this lemma, one gets the elements $u_{i}(5)$ of the piecewise continuous $U$ with $\alpha$ and $\beta$ are defined by:

$$
\left[\begin{array}{c}
\alpha \\
\beta
\end{array}\right]=\Gamma_{n}^{-1}\left[\zeta_{d}-A_{1}^{n} \zeta_{0}\right] .
$$

This control steers subsystem $\Sigma_{1}$ in $n$ sampling periods from the initial state $\zeta_{0}$ to the final state $\zeta_{n}=\zeta_{d}$, i.e. the desired state.

3) Profile of $V$ : The second subsystem can be written as follows:

$$
\begin{aligned}
\Sigma_{2}: \dot{z} & =A_{\Sigma_{2} z+B_{\Sigma_{2}} v} \\
& =\left(\begin{array}{ccccccc}
0 & 1 & & & & & \\
& 0 & u & & & 0 & \\
& & \ddots & & & \\
& & & \ddots & 1 & & \\
& & & & 0 & u & \\
& & & & & 0 & 1 \\
& & & & & 0
\end{array}\right) z+\left(\begin{array}{c}
0 \\
\\
\vdots \\
\\
\\
1
\end{array}\right)
\end{aligned}
$$

with $A_{\Sigma_{2}} \in R^{2 m \times 2 m}$ and $B_{\Sigma_{2}} \in R^{2 m \times 1}$.

We follow the same approach as for the control $U$ however, without any special form. The sampled states of $\Sigma_{2}$ are at each sampling period $\delta$ given by:

$$
\left\{\begin{array}{c}
z_{1}=A_{2_{0}} z_{0}+B_{2_{0}} v_{0} \\
z_{2}=A_{2_{1}} z_{1}+B_{2_{1}} v_{1} \\
\vdots \\
z_{n}=A_{2_{n-1}} z_{n-1}+B_{2_{n-1}} v_{n-1} .
\end{array}\right.
$$

Matrices $A_{2_{i}}$ and $B_{2_{i}}$ are found by sampling $A_{\Sigma_{2}}$ and $B_{\Sigma_{2}}$ with $u=u_{i}$.

$$
\begin{gathered}
A_{2_{i}}=\left[\begin{array}{ccccc}
1 & u_{i} \delta & u_{i}^{2} \frac{\delta^{2}}{2 !} & \ldots & u_{i}^{2 m-1} \frac{\delta^{2 m-1}}{(2 m-1) !} \\
0 & 1 & u_{i} \delta & \ldots & u_{i}^{2 m-2} \frac{\delta^{2 m-2}}{(2 m-2) !} \\
\vdots & & & & \vdots \\
0 & \ldots & 0 & 1 & u_{i} \delta \\
0 & \ldots & 0 & 0 & 1
\end{array}\right] \\
B_{2_{i}}=\left[\begin{array}{l}
u_{i}^{2 m-1} \frac{\delta^{2 m}}{2 m !} \\
u_{i}^{2 m-2} \frac{\delta^{2 m-1}}{(2 m-1) !} \\
\vdots \\
u_{i} \frac{\delta^{2}}{2 !}
\end{array}\right.
\end{gathered}
$$

The final state $z_{n}$ after $n$ sampling periods takes the following form depending on $\Phi_{n}$ and $\Psi_{n}: z_{n}=\Phi_{n} z_{0}+\Psi_{n} V$ where $\Psi_{n}, \Phi_{n}$ and $V$ are given by:

$$
\begin{gathered}
\Psi_{n}=\left[A_{2_{n-1}} A_{2_{n-2}} \cdots A_{2_{1}} B_{2_{0}}, \ldots, A_{2_{n-1}} B_{2_{n-2}}, B_{2_{n-1}}\right] \\
\Phi_{n}=\prod_{j=0}^{n-1} A_{2_{j}} \\
V=\left[\begin{array}{llll}
v_{0} & v_{1} & \ldots & v_{n-1}
\end{array}\right]^{T} .
\end{gathered}
$$


4) Existence of $V$ : To find the elements of $V$ (15), $\Psi_{n}$ must be full rank. The full rank of $\Psi_{n}$ is established by the following lemma.

Lemma 2: If $u \neq 0$, then $\Psi_{n}$ is of full rank for $n>2 m$.

Proof: See appendix II. It is derived essentially from controllability issues.

By applying Lemma 2, the piecewise continuous control $V$ steers $\Sigma_{2}$ from the initial state $z_{0}$ to a state $z_{n}=z_{d}$ in $n$ sampling periods:

$$
V=\Psi_{n}^{+}\left(z_{d}-\Phi_{n} z_{0}\right)
$$

where $\Psi_{n}^{+}$is the pseudo-inverse matrix of $\Psi_{n}$.

$$
\Psi_{n}^{+}=\Psi_{n}^{T}\left(\Psi_{n} \Psi_{n}^{T}\right)^{-1}
$$

The global control obtained, denoted by $W=[U V]^{T}$, steers the system (1) from an initial state $y_{0}$ to any desired final state $y_{d}$ without considering saturation constraints:

$$
-u^{\max } \leq u \leq u^{\max },-v^{\max } \leq v \leq v^{\max } .
$$

The objective of the following section is to develop an algorithm that converges iteratively to an admissible sequence of control (i.e. respects the constraints).

\section{SUB-OPTIMAL SOLUTIONS OF THE OPTIMIZATION PROBLEM}

Our objective here is to find the control inputs, at each sampling period, $u_{i}$ and $v_{i}$ such that saturation constraints (17) are respected. The input of this control algorithm are the initial state $y_{0}$ and desired final state after $n$ sampling periods $y_{n}=y_{d}=\left(\zeta_{d}, z_{d}\right)$. An algorithm $A\left(y_{0}\right)$ based on the following lemma is developed.

Lemma 3: $\lim _{n \rightarrow \infty}\|U\| \rightarrow 0, \lim _{n \rightarrow \infty}\|V\| \rightarrow 0$. Proof: See appendix ??

Remark: This lemma is important as it gives a relationship between horizon length $n$ and the magnitude of the control. If $n$ increases then the magnitude of the elements of $U$ and $V$ will tend to zero.

\section{A. Algorithm $A\left(y_{0}\right)$}

Using the algorithm stated below an admissible control is computed. The algorithm consists of the following steps:

1) Initialize the prediction horizon $n$.

2) Find $\Gamma_{n}, \Phi_{n}$ and $\Psi_{n}$ given by (9), (II-.3) and (15) .

3) Find $U$ and $V$ using (5) et (16).

4) Test the constriants of saturation (17).

5) If step (4) is not respected, increase the horizon length, for example $n=n+1$.

6) Repeat the algorithm from step (2) until the constraints are satisfied.

The result is the admissible control sequences $\hat{W}$ :

$$
\hat{W}=\left(\begin{array}{c}
\hat{U} \\
\hat{V}
\end{array}\right)=\left(\left(\begin{array}{c}
\hat{u}_{0} \\
\hat{v}_{0}
\end{array}\right),\left(\begin{array}{c}
\hat{u}_{1} \\
\hat{v}_{1}
\end{array}\right), \ldots,\left(\begin{array}{c}
\hat{u}_{\hat{n}-1} \\
\hat{v}_{\hat{n}-1}
\end{array}\right)\right)
$$

which will be applied during the time horizon:

$$
\hat{t}_{\hat{n}}\left(y_{0}\right)=\left(\begin{array}{llll}
0 & \delta & \ldots & \hat{n} \delta
\end{array}\right)
$$

where $\hat{n}$ denotes the length of the prediction horizon by applying algorithm $A\left(y_{0}\right)$. The control inputs resulting from the above algorithm provide the necessary control to steer the chained-form system from an initial state $y_{0}$ to the desired final state $y_{\hat{n}}=y_{d}$ in $\hat{n}$ sampling periods while satisfying the saturation constraints. The only limitation of this algorithm occurs when $u$ is zero. In this case, the controllability of the second subsystem $\Sigma_{2}$ is lost hence that of the entire system as well. Discontinuous approaches seek for example to move away from this singularity. From its definition, $u=0$ means that $\alpha$ and $\beta$ of (5) are equal to zero, in other words, equality $\zeta_{d}=\left(A_{1}\right)^{n} \zeta_{0}$ (i.e. 8 ) is satisfied. To avoid this situation, an additional step is applied before executing the algorithm $A\left(y_{0}\right)$. This step also helps to increase the speed of convergence of the state from the initial state $y_{0}$ to the desired final state $y_{d}$. Thus, a constant control $\varepsilon \in\{-1,1\}$ is first applied for 0 to $q_{\max }$ sampling periods:

$$
w_{q, \varepsilon}(\tau)=\left(\begin{array}{c}
\varepsilon u^{\max } \\
0
\end{array}\right) \quad \forall \tau \in[0, q \delta]
$$

The application of this control for $q$ sampling periods directs the system state to an intermediate state $\tilde{y}\left(y_{0}, q, \varepsilon\right)=$ $Y\left(q \delta ; 0 ; y_{0} ; w_{q, \varepsilon}().\right)$. Then, the system is oriented to a desired final state $y_{n}=y_{d}$ by the application of Algorithm $A(\tilde{y})$.

As a result, we obtain a set of control sequences that can steer the system in a different time duration as a function of $q$ and $\varepsilon$. For example, in the case where $q=q_{\max }$ and $\varepsilon=-1$ the control steers the system in a time duration of $q_{\max } \delta+\hat{t}_{\hat{n}}\left(\tilde{y}\left(y_{0}, q_{\max },-1\right)\right)$ where $\hat{t}_{\hat{n}}\left(\tilde{y}\left(y_{0}, q_{\max },-1\right)\right)$ is obtained by the application of $A\left(\tilde{y}\left(y_{0}, q=q_{\max }, \varepsilon=-1\right)\right)$. Since our objective is to steer the system in minimum time then the control sequence with the smallest time duration is selected. Therefore, the objective is to find the optimal and admissible open loop control $w^{o p t}\left(y_{0}\right)$ corresponding to the solution of the following optimization problem

$$
\left(\hat{q}\left(y_{0}\right), \hat{\varepsilon}\left(y_{0}\right)\right)=\operatorname{Arg} \min _{(q, \varepsilon) \in \mathcal{A}\left(y_{0}\right)} q \delta+\hat{t}_{\hat{n}}\left(y_{f}\left(y_{0}, q, \varepsilon\right)\right)
$$

with

$$
\begin{array}{r}
\mathcal{A}\left(y_{0}\right)=\left\{(q, \varepsilon) \in\left\{0, \ldots, q_{\max }\right\} \times\{-1,1\} \mid\right. \\
\left.\hat{t}_{\hat{n}}\left(y_{f}\left(y_{0}, q, \varepsilon\right)\right)<\hat{t}_{\hat{n}}\left(y_{0}\right)-q . \delta\right\} .
\end{array}
$$

The control $w^{o p t}\left(y_{0}\right)$ takes the following form

$w^{o p t}\left(y_{0}\right)=\left[\begin{array}{l}u^{o p t}\left(y_{0}\right) \\ v^{o p t}\left(x_{0}\right)\end{array}\right]=\left(w_{0}^{o p t}, \ldots, w_{\hat{q}\left(y_{0}\right)}^{o p t}, \hat{W}\left(y_{f}\right)\right)$

where $\hat{W}\left(y_{f}\right)$ is the control obtained after the execution of $A\left(y_{f}\right)$. For $j=1, \ldots, \hat{q}\left(y_{0}\right)$ :

$$
w_{j}^{o p t}=\left(\begin{array}{l}
\hat{\varepsilon}\left(y_{0}\right) u^{\max } \\
0
\end{array}\right) .
$$

This control will be applied for the following time horizon:

$$
t^{o p t}\left(y_{0}\right)=\left(0, \delta, \ldots, \delta \hat{q}\left(y_{0}\right), \hat{t}_{\hat{n}}\left(y_{f}\right)\right)
$$

with

$$
\hat{t}_{\hat{n}}\left(y_{f}\right)=\left(\left(\hat{q}\left(y_{0}\right)+1\right) \delta, \ldots,\left(\hat{q}\left(y_{0}\right)+\hat{n}\left(y_{0}\right)\right) \delta\right)
$$




\section{B. Algorithm $B\left(y_{0}\right)$}

This algorithm is applied to find the suboptimal control inputs in open loop that steers system (1) from initial state $y_{0}$ to final desired state $y_{d}$ while respecting the saturation constraints on the control inputs (17).

1) Decompose the system (1) into $\Sigma_{1}$ and $\Sigma_{2}$.

2) For $n, q \leq q_{\max }$ and $\varepsilon= \pm 1$, find the control by applying a constant control plus the algorithm $A\left(y_{0}\right)$.

3) Take the control that corresponds to the solution of the optimization problem (21).

It may be noted that there is no special treatment for the singular case in the optimization problem. In fact, there is no optimization but merely a choice in a finite set of solutions. In the next section, the state feedback control is defined.

\section{STATE FEEDBACK CONTROL}

Using the principles of predictive control, control is achieved by the following state feedback

Theorem 1: The discrete time state feedback defined for $\sigma \in[0, \delta[:$

$$
w(k \delta+\sigma)=w_{1}^{o p t}\left(y_{k}\right)
$$

where $w_{1}^{o p t}\left(y_{k}\right)$ is the first element of the open loop control sequence (Algorithm $B\left(y_{0}\right)$ of section III-B), steers system (1) starting from an initial state $y_{0}$ to a desired final state $y_{d}$.

Proof: See appendix III.

\section{Simulations}

To show the effectiveness of the proposed controller, several simulation studies are carried out on system 1 with $m=3$. Starting from an initial state $x_{0}=$ $[-1.5,0,1.75,0,0.5,0,2,0]^{T}$ under the control inputs $[u, v]$ constrained by $\left[u^{\max }, v^{\max }\right]=[5,5]$ with $q_{\max }=2$ and $\delta=0.1$, we have studied the following cases :

1) Convergence to a final desired state $x_{d}=$ $[0,0,0,0,0,0,0,0]^{T}$ (Figs. 1 and 2),

2) Convergence to null desired state (Figs. 3 and 4 ) from a singular initial state $x_{0}=[0,0,1.75,0,0.5,0,2,0]^{T}$,

3) Convergence to a final desired state $x_{d}$ different from zero $x_{d}=[1,0,0.2,0,-0.3,0,0.1,0]^{T} 1$.

These simulation results show that the proposed controller is able to steer the extended chained form system from any initial state to any desired state. In addition, we have tested the admissible domain of the control with $\left[u^{\max }, v^{\max }\right]=$ $[0.05,0.05]$ and the desired state is the origin $x_{d}=$ $[0,0,0,0,0,0,0,0]^{T}$. These constraints will make the control law converge to a bang-bang type behavior, well known in time optimal control. The effect of the sampling period $\delta$ on the control performance is examined where $\delta$ is equal to 1 sec instead of $0.1 \mathrm{sec}$. We have noticed that the resulting closed-loop convergence time increases with the increase of the sampling period. To end, the effect of the value of $q_{\max }$ on the convergence time is also studied where we $q_{\max }=10$ is used instead of $q_{\max }=2$. By increasing $q_{\max }$, the control algorithms generate more trajectories potentially with smaller convergence time.

\footnotetext{
${ }^{1}$ Figures are omitted due to page limitations.
}

\section{CONCLUSION}

In this paper, a state feedback control which assures the convergence of the extended chained form system to any position is proposed. This controller is based on model predictive control and handles the singular situations without any special treatment. It also respects arbitrary saturation constraints on the control inputs. The proposed scheme is real-time implementable. The average calculation time for each iteration of the previous simulations uses $0.01 \mathrm{sec}$ under MATLAB $^{\circledR}$. This time can be reduced by a factor of 10 to 100 using a $\mathrm{C}$ framework.

\section{APPENDIX I EXISTENCE OF $U$}

Lemma $\forall n>1, \Gamma_{n}$ is full rank.

Proof: Matrix $\Gamma_{n}$ can be written as:

$\left[\begin{array}{cc}\sum_{i=0}^{n-1}\left[\frac{\delta^{2}}{2}+(n-1-i) \delta^{2}\right] i & \sum_{i=0}^{n-1} \frac{\delta^{2}}{2}+(n-1-i) \delta^{2} \\ \sum_{i=0}^{n-1} i \delta & \sum_{i=0}^{n-1} \delta\end{array}\right]$

The development of the four elements of (26) gives:

$$
\begin{aligned}
& \Gamma_{n}^{11}=\frac{\delta^{2} n(n-1)\left(n-\frac{1}{2}\right)}{2}-\delta^{2} \sum_{i=0}^{n-1} i^{2} \\
& \Gamma_{n}^{12}=\frac{\delta^{2} n^{2}}{2} \\
& \Gamma_{n}^{21}=\frac{\delta n(n-1)}{2} \\
& \Gamma_{n}^{22}=\delta n
\end{aligned}
$$

Thanks to (29), $\Gamma_{n}$ is full rank when $\Gamma_{n}^{11} \neq \frac{n-1}{2} \Gamma_{n}^{12}$. The proof is based on the following difference $\Gamma_{n}^{11}-\frac{n-1}{2} \Gamma_{n}^{12}$,

$$
\Gamma_{n}^{11}-\frac{n-1}{2} \Gamma_{n}^{12}=-\delta^{2} \sum_{i=0}^{n-1} i^{2}+\frac{n \delta^{2}}{4}(n-1)^{2}
$$

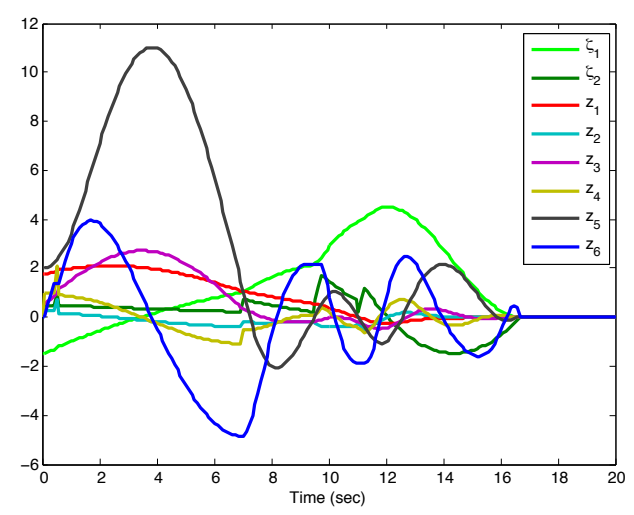

Fig. 1. The convergence of the extended chained form system starting from the initial state $x_{0}=[-1.5,0,1.75,0,0.5,0,2,0]^{T}$ and converging to a final desired state $x_{d}=[0,0,0,0,0,0,0,0]^{T}$ with the saturation constraints on the control inputs $\left[u^{\max }, v^{\max }\right]=[5,5]$ and $q_{\max }=2$ with $\delta=0.1 \mathrm{sec}$. 


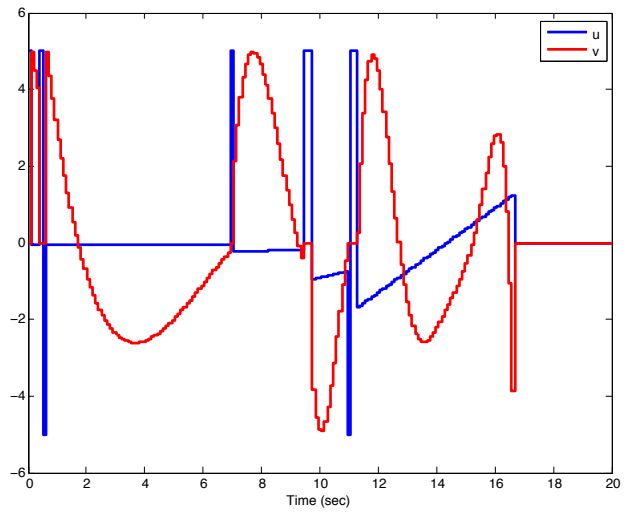

Fig. 2. The control inputs $u$ (in blue) and $v$ (in red) applied for steering the extended chained form system from the initial state $x_{0}=[-1.5,0,1.75,0,0.5,0,2,0]^{T}$ to a final desired state $x_{d}=$ $[0,0,0,0,0,0,0,0]^{T}$ with the saturation constraints on the control inputs $\left[u^{\max }, v^{\max }\right]=[5,5]$ and $q_{\max }=2$ with $\delta=0.1 \mathrm{sec}$.

For $I_{n}:=\sum_{i=0}^{n} i^{2}$ et $J_{n}:=\frac{(n+1) n^{2}}{4}$, then $\Gamma_{n}^{11}-\frac{n-1}{2} \Gamma_{n}^{12}=$ $\delta^{2}\left(J_{n-1}-I_{n-1}\right)$. One also has

$$
\begin{aligned}
\Delta I_{n} & =I_{n}-I_{n-1}=n^{2} \\
\Delta J_{n} & =J_{n}-J_{n-1}=\frac{3 n^{2}}{4}-\frac{n}{4}
\end{aligned}
$$

It is obvious that $\Delta I_{n}>\Delta J_{n}$ and since $I_{1}>J_{1}$ one has that $\forall n>0, I_{n}>J_{n}$. Therefore, $\Gamma_{n}^{11} \neq \frac{n-1}{2} \Gamma_{n}^{12}$ and $\Gamma_{n}$ is full rank $\forall n>1$.

\section{APPENDIX II \\ EXISTENCE OF $V$}

We find a relation between the length of the prediction horizon $n$ and system's dimension $m$ such that matrix $\Psi_{n}$ is full rank.

Lemma If $u \neq 0$, then $\Psi_{n}$ is of full rank for $n>2 m$. Proof: To prove this lemma, we have to use the following relationship ( $\alpha=\beta=0 \leftrightarrow \zeta_{d}=\zeta_{0}$ ). This relationship means that the command $u$ vanishes only when the desired state is equal to the initial state. Assume first that $\alpha=\beta=0$, then necessarily and thanks to (??), we have the following relation between the initial state and the desired state:

$$
\zeta_{d}=A_{1}^{n} \zeta_{0}=A_{1}^{n}\left(\begin{array}{c}
\zeta_{0}^{1} \\
\zeta_{0}^{2}
\end{array}\right) \quad \text { with } \quad A_{1}=\left(\begin{array}{ll}
1 & \delta \\
0 & 1
\end{array}\right)
$$

The desired state $\zeta_{d}(34)$ is written:

$$
\zeta_{d}=\left(\begin{array}{c}
\zeta_{d}^{1} \\
\zeta_{d}^{2}
\end{array}\right)=\left(\begin{array}{c}
\zeta_{0}^{1}+n \delta \zeta_{0}^{2} \\
\zeta_{0}^{2}
\end{array}\right)
$$

Since the position convergence is considered, $\zeta_{d}$ is of the form $\left[\begin{array}{ll}\zeta_{d}^{1} & 0\end{array}\right]^{T}$ where $\zeta_{d}^{1}$ is the desired target position with zero speed $\zeta_{d}^{2}=0$. Therefore, we obtain the desired state equal to the initial state $\left(\zeta_{0}^{2}=\zeta_{d}^{2}=0\right.$ et $\left.\zeta_{0}^{1}=\zeta_{d}^{1}\right)$. In the case where the initial state is different from the desired state $\left(\zeta_{0} \neq \zeta_{d}\right)$, then one has $\alpha \neq 0$ or $\beta \neq 0$. The question is whether $\alpha$ and $\beta$ are at the same time different from zero. The answer is based on the characteristics of the $u_{i}$. We know that $u_{i}$ can be canceled only once during the horizon $n$ because it takes the form of a piecewise continuous straight line. Indeed, if there are two different time instants $i_{1}$ and $i_{2}\left(i_{1} \neq i_{2}\right)$ such that the corresponding commands $u_{i_{1}}$ and $u_{i_{2}}$ vanish $\left(u_{i_{1}}=u_{i_{2}}=0\right)$, then applying the definition of $\alpha$ and $\beta$ (5) we obtain the following system of equations:

$$
\left\{\begin{array}{l}
\alpha i_{1}+\beta=0 \\
\alpha i_{2}+\beta=0
\end{array} .\right.
$$

In this case $\alpha$ and $\beta$ vanish. This is a contradiction because we have $\alpha \neq 0$ or $\beta \neq 0$. Control $u_{i}$ then vanishes only once during the horizon length $n$ and the two time instants $i_{1}$ and $i_{2}$ are equal $\left(i_{1}=i_{2}\right)$. Hence, the parameters $\alpha$ and $\beta$ are equal to zero if and only if the initial state is identical to the desired state $\zeta_{d}=\zeta_{0}$. We are now sure that if an initial state is different from the desired state, one element of $U$ can be canceled.

The dimension of matrix $\Psi_{n}$ is clearly $2 m \times n$. If we choose the horizon length $n$ two times larger than the dimension of $\Sigma_{2}(n>2 m)$, we can ensure that there is at least $2 m$ time periods where $u_{i} \neq 0$. In this case we can guarantee the existence of at least $2 m$ controllable pairs $\left[A_{2 i}, B_{2 i}\right]$ of the matrix $\Psi_{n}$. So we can conclude that $\Psi_{n}$ is full rank if $\zeta_{d} \neq \zeta_{0}$ and $n>2 m$.

\section{APPENDIX III \\ PROOF OF THE MAIN RESULT}

In order to state the main result, we need the following proposition

Proposition 1: For $\delta$ positive and constant there exists a scalar function $L: \mathbb{R}^{n} \rightarrow \mathbb{R}^{+}$with the following properties

1) $(L(y)=0) \Leftrightarrow\left(y=y_{d}\right)$.

2) $L$ is radially unbounded.

3) $\forall k \in \mathbb{N}$

$$
L\left(y_{c l}(k+1)\right)-L\left(y_{c l}(k)\right) \leq-\delta \text { once } L\left(y_{c l}(k)\right)>\delta
$$

The state $y_{c l}($.$) denotes the trajectories in closed-loop by$ applying the control of theorem 1 .

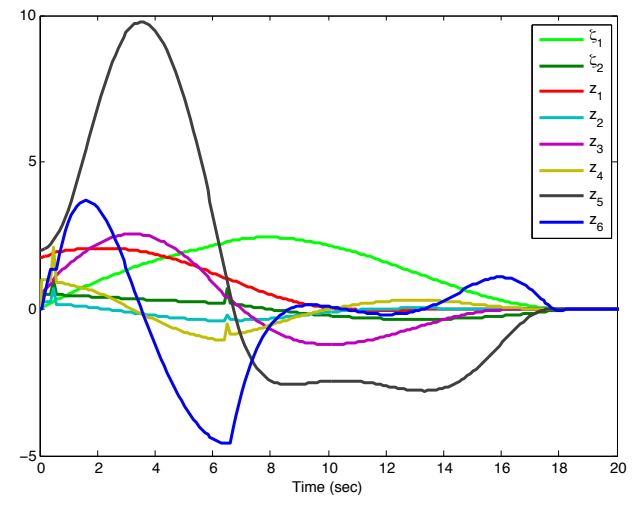

Fig. 3. The convergence of the extended chained form system starting from a singular initial state $x_{0}=[0,0,1.75,0,0.5,0,2,0]^{\mathbf{T}}$ to a final desired state $x_{d}=[0,0,0,0,0,0,0,0]^{T}$ with the saturation constraints on the control inputs $\left[u^{\max }, v^{\max }\right]=[5,5]$ and $q_{\max }=2$ with $\delta=0.1$ sec. 


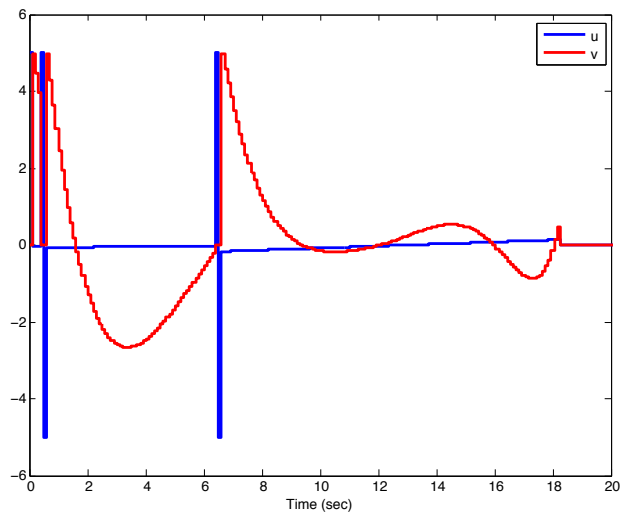

Fig. 4. The control inputs $u$ (in blue) and $v$ (in red) applied for steering the extended chained form system from a singular initial state $\mathbf{x}_{\mathbf{0}}=[\mathbf{0}, \mathbf{0}, \mathbf{1 . 7 5}, \mathbf{0}, \mathbf{0 . 5}, \mathbf{0}, \mathbf{2}, \mathbf{0}]^{\mathbf{T}}$ to a final desired state $x_{d}=$ $[0,0,0,0,0,0,0,0]^{T}$ with the saturation constraints on the control inputs $\left[u^{\max }, v^{\max }\right]=[5,5]$ and $q_{\max }=2$ with $\delta=0.1 \mathrm{sec}$.

Proof: In our case, it will be shown that $L$ defined by

$$
\begin{array}{r}
L(y)=\min _{i \in\{1, \ldots, n\}}\left\{t_{i}^{o p t}(y) \mid\right. \\
\left.X\left(t_{i}^{o p t}(y) ; 0 ; x ; W\left(., t^{o p t}\left(y_{0}\right), w^{o p t}\left(y_{0}\right)\right)\right)=0\right\}
\end{array}
$$

satisfies proposition 1. Note that from the definitions of $\left(t^{o p t}(y), w^{o p t}(y)\right)$ (equations (22) and (23)), we know that $L(y)$ is well defined such that $L(y) \leq t_{n}^{\text {opt }}(y)$. The characteristics of $L(y)$ of Proposition 1 are proved as follows:

1) $(L(y)=0) \Leftrightarrow\left(y=y_{d}\right)$ results directly of the fact that under bounded control, the system state cannot be oriented from $y_{0} \neq y_{d}$ to $y_{d}$ infinitely fast.

2) $L$ is radially unbounded results from the fact that under bounded control, the necessary time to steer the state from $y_{0}$ to $y_{d}$ tends to $\infty$ when $\left\|y_{0}\right\|$ tends to $\infty$.

3) We use the solution of the optimization problem

$$
\left(\hat{q}\left(y_{0}\right), \hat{\varepsilon}\left(y_{0}\right)\right)=\operatorname{Arg} \min _{(q, \varepsilon) \in \mathcal{A}\left(x_{0}\right)} q \delta+\hat{t}_{\hat{n}}\left(y_{f}\left(y_{0}, q, \varepsilon\right)\right)
$$

and as a cost function

$$
J\left(q, \varepsilon, y_{0}\right)=q \delta+\hat{t}_{n}\left(y_{f}\left(y_{0}, q, \varepsilon\right)\right)
$$

We use $\left(\hat{q}_{k}, \hat{\varepsilon}_{k}\right)$ to denote $\left(\hat{q}\left(y_{c l}(k)\right), \hat{\varepsilon}\left(y_{c l}(k)\right)\right)$. Two cases must be considered:

Case 1: In this case where $\hat{q}_{k}>0$, we define

$$
y^{+}(k):=X\left(\hat{q}_{k} \delta ; 0 ; y_{c l}(k) ; u_{\hat{q}_{k}, \hat{\varepsilon}_{k}}(.)\right)
$$

Par definition, $L\left(y_{c l}(k)\right)$ is given by:

$$
L\left(y_{c l}(k)\right)=\hat{q}_{k} \delta+\hat{t}_{i_{0}}\left(y^{+}(k)\right) \text { for } i_{0} \leq n
$$

Knowing that

$$
X\left(\tilde{q}_{k+1} \delta ; 0 ; y_{c l}(k+1) ; w_{\tilde{q}_{k+1}, \tilde{\varepsilon}_{k+1}}(.)\right)=y^{+}(k)
$$

with the suboptimal solutions $\left(\tilde{q}_{k+1}, \tilde{\varepsilon}_{k+1}\right)=\left(\hat{q}_{k}-\right.$ $\left.1, \hat{\varepsilon}_{k}\right)$. Therefore, with (39)

$$
\begin{aligned}
L\left(y_{c l}(k+1)\right) & \leq J\left(\tilde{q}_{k+1}, \tilde{\varepsilon}_{k+1}, y_{c l}(k+1)\right) \\
& \left.\leq\left(\hat{q}_{k}-1\right) \delta+\hat{t}_{i_{0}}\left(y^{+}(k)\right)\right) \\
& \leq L\left(y_{c l}(k)\right)-\delta
\end{aligned}
$$

The inequality (37) is then verified.

Case 2: In the case where $\hat{q}_{k}=0$, the next state of the closed-loop trajectory is clearly given by

$y_{c l}(k+1)=X\left(\delta ; 0 ; y_{c l}(k) ; W\left(0, t^{o p t}\left(y_{k}\right), w_{0}^{o p t}\left(y_{k}\right)\right)\right)$

Now one can see that choosing the suboptimal solution $\left(\tilde{q}_{k+1}, \tilde{\varepsilon}_{k+1}\right)=\left(0, \hat{\varepsilon}_{k}\right)$ implies that:

$$
J\left(\tilde{q}_{k+1}, \tilde{\varepsilon}_{k+1}, y_{c l}(k+1)\right)=\hat{t}_{n}\left(y_{c l}(k+1)\right)
$$

But one has

$$
\hat{t}_{n}\left(y_{c l}(k+1)\right)=\hat{t}_{n}\left(y_{c l}(k)\right)-\delta
$$

This with (41) prove that in all the cases where $L\left(x_{c l}(k)\right)>\delta$, the necessary time to go from $y_{c l}(k+1)$ to $y_{d}$ is less by at least one period $\delta$ the time required to go from $y_{c l}(k)$ to $y_{d}$. Here again inequality (37) is verified.

\section{REFERENCES}

[1] M. Alamir and N. Marchand. Constrained minimum-time-oriented feedback control for the stabilization of nonholonomic systems in chained form. Journal of Optimization Theory and Applications, 118(2):229-244, 2003.

[2] N.P.I. Aneke, D.A Lizarraga, and H. Nijmeijer. Homogeneous stabilization of the extended chained form system. In IFAC 15th Triennial World Congress, Barcelona, Spain, 2002.

[3] R.W. Brockett. Differential Geometric Control Theory, chapter Asymptotic stability and feedback stabilization, pages 181-191. University of Michigan Press, Ann Arbor, Michigan, 1983.

[4] S. S. Ge, Z. Sun, T. H. Lee, and M. W. Spong. Feedback linearization and stabilization of second-order non-holonomic chained systems. 74(14):1383-1392, 2001.

[5] A. Hably, N. Marchand, and M. Alamir. Constrained minimum time oriented stabilization of extended chained form systems. In The joint IEEE Conf. on Decision and Control and European Control Conference, 2005.

[6] J. Hauser, S. Sastry, and Meyer G. Non linear control design for slightly nonminimum phase systems: Application to v/stol aircraft. Automatica, 28:665-679, 1992.

[7] M.C Laiou and A. Astolfi. Discontinuous control of high-order generalized chained system. Systems \& Control letters, 37:309-322, 1999.

[8] D.A Lizarraga and J. Sosa. Vertically transverse functions as an extension of the transverse function control approach for secondorder systems. In $44^{\text {th }}$ IEEE conf. on Decision and Control and the European Control Conference 2005, CDC-ECC'05, 2005.

[9] J. Luo and P. Tsiotras. Control design for chained-form systems with bounded inputs. 39(2):123-131, Feb. 2000.

[10] P. Morin and C. Samson. Control of underactuated mechanical systems by the transverse function approach. In $44^{\text {th }}$ IEEE conf. on Decision and Control and the European Control Conference 2005, CDC-ECC'05, 2005.

[11] R.M. Murray and S. Sastry. Nonholonomic motion planning: Steering using sinusoids. IEEE Transactions on Automatic Control, 38,:700716, 1993.

[12] Y. P. Tian and S. Li. Exponential stabilization of nonholonomic dynamic systems by smooth time-varying control. Automatica, 38:11391146, 2002.

[13] M. Yamada, S. Ohta, and Y. Funahashi. State and output feedback stabilization for second-order nonholonomic chained systems based on sampled data control approach. In American-Control-Conference 2003, ACC'03, 2003. 